LAWRENCE LIVERMORE N A T IO N A L LABORATORY

Functional One-Dimensional

Lipid Bilayers on Carbon

Nanotube Templates

Alexander Artyukhin, Alexei Shestakov, Jennifer Harper, Olgica Bakajin, Pieter Stroeve, Aleksandr Noy

August 10, 2004

Science 
This document was prepared as an account of work sponsored by an agency of the United States Government. Neither the United States Government nor the University of California nor any of their employees, makes any warranty, express or implied, or assumes any legal liability or responsibility for the accuracy, completeness, or usefulness of any information, apparatus, product, or process disclosed, or represents that its use would not infringe privately owned rights. Reference herein to any specific commercial product, process, or service by trade name, trademark, manufacturer, or otherwise, does not necessarily constitute or imply its endorsement, recommendation, or favoring by the United States Government or the University of California. The views and opinions of authors expressed herein do not necessarily state or reflect those of the United States Government or the University of California, and shall not be used for advertising or product endorsement purposes. 


\title{
Functional one-dimensional lipid bilayers on carbon nanotube templates
}

\author{
Alexander B. Artyukhin, ${ }^{1,3}$ Aleksei Shestakov, ${ }^{2}$ Jennifer Harper, ${ }^{1}$ \\ Olgica Bakajin, ${ }^{1}$ Pieter Stroeve, ${ }^{3}$ and Aleksandr Noy ${ }^{1, *}$ \\ ${ }^{1}$ Biosecurity and Nanosciences Laboratory, Lawrence Livermore National Laboratory, \\ 7000 East Ave, Livermore, CA 94550 \\ ${ }^{2}$ AX Division, Lawrence Livermore National Laboratory, \\ 7000 East Ave, Livermore, CA 94550 \\ ${ }^{3}$ Department of Chemical Engineering and Materials Science, \\ University of California Davis, Davis, CA 95616
}

*To whom correspondence should be addressed; E-mail: noy1@1lnl.gov.

\begin{abstract}
We present one-dimensional (1-D) lipid bilayer structures that integrate carbon nanotubes with a key biological environment- phospholipid membrane. Our structures consist of lipid bilayers wrapped around carbon nanotubes modified with a hydrophilic polymer cushion layer. Despite high bilayer curvature, the lipid membrane maintains its fluidity and can sustain repeated damage-recovery cycles. We also present the first evidence of spontaneous insertion of pore-forming proteins into 1-D lipid bilayers. These structures could lead to the development of new classes of biosensors and bioelectronic devices.
\end{abstract}

Carbon nanotubes have catalyzed many important discoveries in physics, chemistry and materials science $(1,2)$. To achieve a similar success with biophysical applications, such as biosensing (3), intracellular probing (4), and bio-NEMS (5), we must overcome the challenge of integrating nanotubes with key biological environments. Researchers have demonstrated noncovalent modification of nanotubes with proteins (3), specific peptides (6), DNA sequences (7), 
and monolayers of amphiphilic molecules (8). However, many vital biological proteins function only in a bilayer membrane environment, and current approaches do not provide a viable route for functional coupling of membrane proteins to the nanotube surface.

We demonstrate a new way of functionalizing single wall carbon nanotube surface with a phospholipid bilayer, which is a main component of biological membranes. In our experiments, the nanotube surface, modified with hydrophilic polymer layers, serves as a template for assembly of the lipid bilayer, which forms a continuous shell around the nanotube. The key difference between our approach and the previous attempts to modify the nanotube surface is that our supported bilayers maintain the key properties of biological membranes: the lateral mobility of lipid molecules and the ability to incorporate membrane proteins. Our method provides a robust and general strategy for achieving biocompatibility of carbon nanotubes.

Direct assembly of lipid bilayers on carbon nanotubes is challenging. Typical diameters of single wall carbon nanotubes range from 1 to 5 nanometers, which is well below the smallest reported curvature of a lipid bilayer (9). More important, hydrophobic nanotube surface does not support the polar outer groups of a lipid bilayer; instead, it produces a monolayer of lipids with hydrophobic tails in direct contact with the nanotube $(8,10)$. To support a lipid bilayer, the nanotube surface must bear a hydrophilic cushion layer. Recently we demonstrated controlled modification of nanotube surfaces using layer-by-layer self-assembly of polyelectrolytes (11). The resulting multilayer polymer structure is ideal for providing the hydrophilic cushion, because ionic polymer support stabilizes the oppositely charged bilayers (12-14). In addition, the number of polymer layers controls the polymer cushion thickness, which allows us to match the diameter of the support to the critical curvature of the lipid bilayer.

We start our assembly process (Fig. 1) by modifying single wall carbon nanotubes suspended across the empty holes of a TEM grid with several alternating polymer layers composed of strong polyelectrolytes, such as poly(diallyldimethylammonium chloride) (PDDA), 


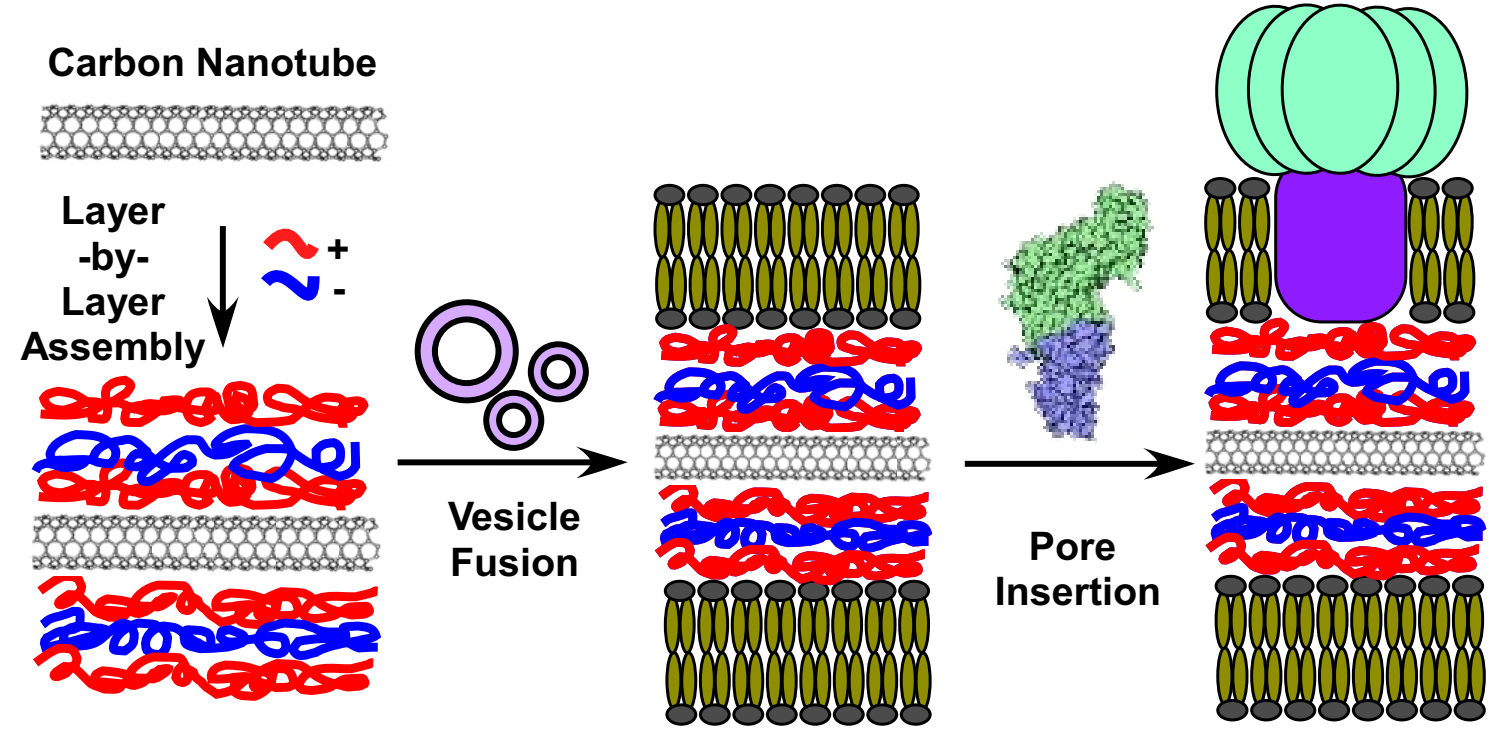

Fig. 1: Schematics of 1-D bilayer assembly process. First, carbon nanotubes are coated with alternating layers of oppositely charged polyelectrolytes (red and blue), followed by the formation of lipid bilayer (black and brown) by vesicle fusion. Additional exposure to the solution of monomeric bacterial toxin (PA63 fragment of the anthrax toxin shown) results in the formation of the oligomeric protein pore in the bilayer membrane. 
polystyrene sulfonate (PSS), and poly(allylamine hydrochloride) (PAH) (15). Suspending the nanotubes between solid supports allowed us to form continuous coating around their circumference. TEM images show that polymer layers produce generally smooth continuous coating on the nanotubes over large distances (Fig. 2A-E), with the diameter of the final structure of 10-15 nm (16). We then created supported lipid bilayers on our "cushioned" carbon nanotubes using vesicle fusion (17). To enable visualization of the final structure our vesicles incorporated a small fraction of a fluorescent dye (15). Scanning confocal fluorescent microscopy images of the resulting structures show linear fluorescent features inside the holes of the TEM grid (Fig. 2F-I), which correspond to the lipid-coated carbon nanotubes stretching across the grid holes. These results indicate that partial strain relief in the direction of the nanotube axis coupled with the electrostatic attraction of the bilayer to the oppositely charged polymer support is sufficient to drive the fusion process and stabilize the bilayer in 1-D configuration. We also argue that incorporation of charged lipids in the membrane should reject subsequent lipid layer assembly and formation of multilayers. Zhang et al. have observed similar behavior for bilayers adsorbed on flat substrates (18); therefore we assume that our experiments produced a single bilayer on polymer-coated carbon nanotubes.

One of the most important features of a functional lipid bilayer is the ability of the lipid molecules to diffuse along the bilayer plane. To prove that our bilayers keep this property and to verify the bilayer continuity we used fluorescence recovery after photobleaching (FRAP) to study mobility of the lipid molecules (19). A large percentage of our 1-D lipid bilayers showed recovery of the fluorescence signal in the range of $20-90 \%$, often sustaining multiple bleachrecovery cycles in the same spot (Fig. 3A,B). Fig. 3A presents an example of two such cycles, which show recovery to an almost identical level. Some lipid-coated nanotubes were even able to sustain recovery over 10 bleaching cycles (Fig. 3B). These results demonstrate that the lipid coating on carbon nanotubes is continuous, with the lipid molecules keeping their mobility even 

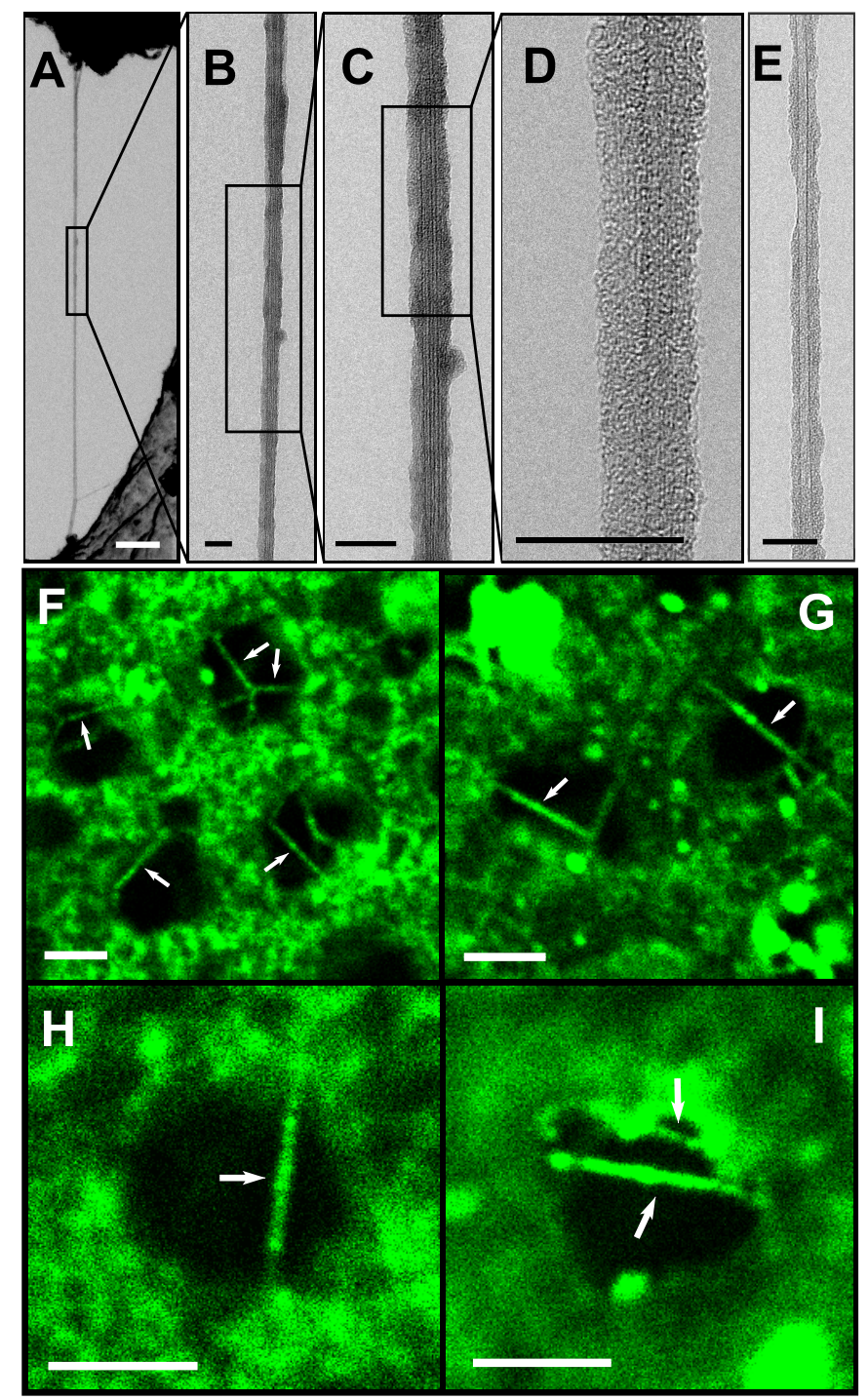

Fig. 2: (A-E) TEM images (300 kV) of a small bundle (A-D) and individual (E) carbon nanotubes coated with 5 alternating PAH/PSS layers. (F-I) Fluorescent images (ex. $488 \mathrm{~nm}$, em. $520 \mathrm{~nm}$ ) of carbon nanotubes coated with 5 PAH/PSS (F-H) or PDDA/PSS (I) layers and a lipid bilayer. The bilayer is composed of $75 \%$ anionic lipid (1-stearoyl-2-oleoylphosphatidylserine, SOPS), 23\% zwitterionic lipid (1-palmitoyl-2-oleoylphosphatidylcholine, POPC), and 2\% fluorescent probe (BODIPY-PC) (15). For fluorescent imaging we placed a TEM grid with lipid functionalized nanotubes in a thin water layer between two glass cover slips. Nanotubes coated with the bilayer are indicated with arrows. Scale bars: $200 \mathrm{~nm}(\mathrm{~A}), 20 \mathrm{~nm}(\mathrm{~B}-\mathrm{E}), 5 \mu \mathrm{m}(\mathrm{F}-\mathrm{I})$. 
in these high-curvature structures.

We can further characterize lipid mobility in 1-D bilayers by using a quantitative model of the bleaching and recovery process. A rigorous model of this process must consider diffusion in a tubular structure. We note that in our experiments the size of the bleaching spot (ca. 500 $\mathrm{nm}$ ) is much larger than the lipid tubule diameter; therefore we can assume that the laser beam bleaches the front and back side of the nanotube uniformly. In this case we can use a simple 1-D diffusion model (Fig. 3C). The normalized concentration of mobile fluorescent lipid molecules along the nanotube, $u(x, t)$, can be then described by the following diffusion equation, where the first term describes diffusion and the second term describes photobleaching:

$$
\partial_{t} u=\partial_{x}\left(D \partial_{x} u\right)-R(x, t) u
$$

Our model also assumes that initially the lipid is distributed uniformly along the nanotube:

$$
\left.u\right|_{t=0}=1
$$

The boundary conditions describe lipid molecules behavior at the nanotube junction with the substrate support. Fluorescence images shown on the Fig. 2F-I indicate that lipid coverage extends onto the TEM grid support. We assume that the bilayer on the grid can act as a reservoir to support the recovery of the bilayer on the nanotube (20), leading to the Dirichlet boundary condition:

$$
\left.u\right|_{(x=0, X)}=u_{b}
$$

Experimentally-observed fluorescence is then proportional to the total amount of fluorescent molecules within the collection spot, from $x_{l 0}$ to $x_{l 1}$ : 

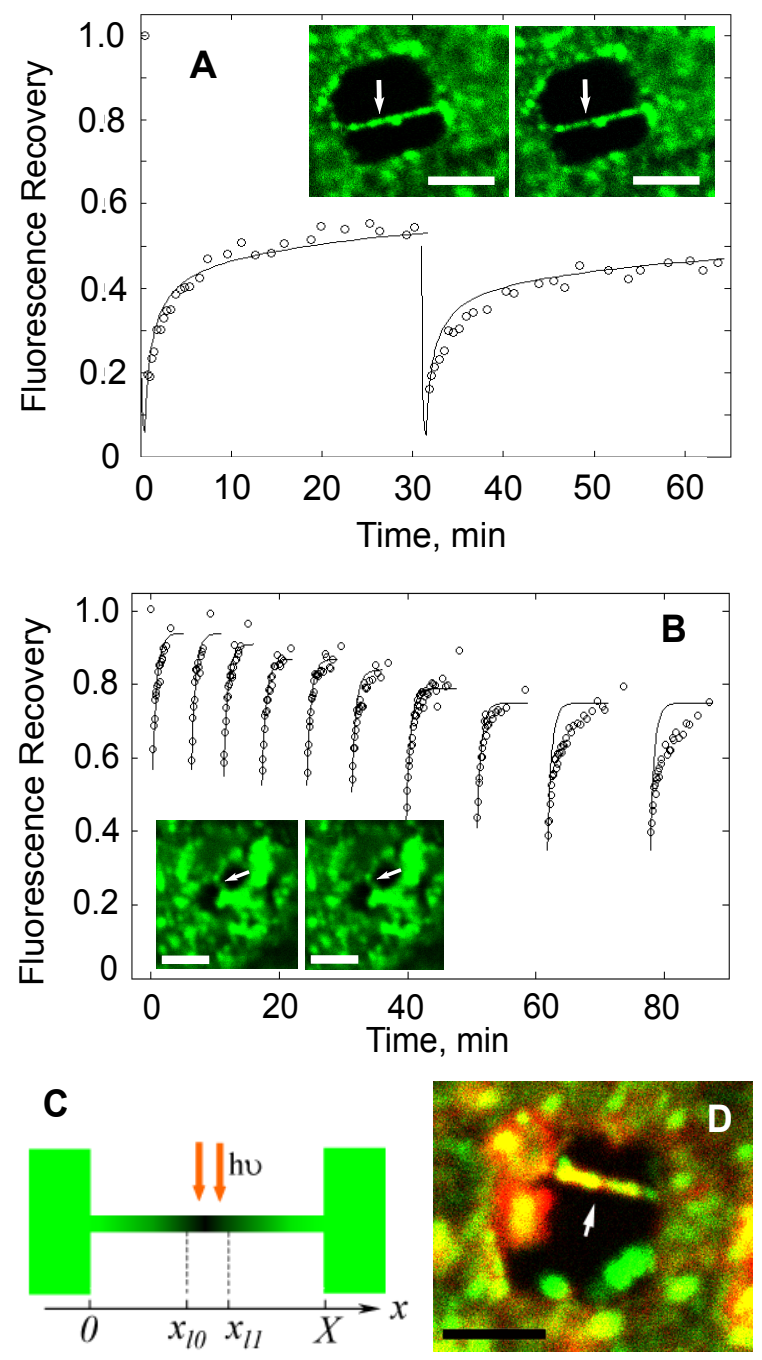

Fig. 3: (A,B) Experimental (dots) and model (lines) fluorescence recovery curves during 2 (A) and 10 (B) FRAP cycles on the same spot on lipid-coated nanotubes. The polymer "cushion" is composed of PDDA and PSS. Parameters of the fit: (A) bleaching time $\left(t_{l}\right) 30 \mathrm{~s}$, bleaching rate $(R) 0.25 \mathrm{~s}^{-1}$, nanotube length $(X) 7 \mu \mathrm{m}$, diffusion coefficient $(D) 4 \cdot 10^{-3} \mu^{2} / \mathrm{s}$. (B) $t_{l}=30 \mathrm{~s}$ in first 6 cycles, $1 \mathrm{~min}$ in $7^{\text {th }}$ and $8^{\text {th }}$ cycles, $2 \mathrm{~min}$ in $9^{\text {th }}$ and $10^{\text {th }}$ cycles, $R=0.1 \mathrm{~s}^{-1}, X=0.8 \mu \mathrm{m}$, $D=2 \cdot 10^{-3} \mu^{2} / \mathrm{s}$. Left (right) insets on A and B show fluorescent images of the corresponding nanotubes before first bleaching (after last recovery). Nanotubes are indicated with arrows. (C) Schematics of the diffusion model. Lipid-coated nanotube is attached to the substrate that can serve as a reservoir of lipid molecules at two points. The bleaching laser spot is focused in the middle of the nanotube. (D) Combined two-color fluorescent image of a lipid-coated nanotube after PA63 adsorption. Green color corresponds to lipid (ex. $488 \mathrm{~nm}$, em. $520 \mathrm{~nm}$ ), red color (ex. $633 \mathrm{~nm}$, em. $650 \mathrm{~nm}$ ) corresponds to the protein. Yellow color corresponds to the areas of colocalization of lipid and protein. Scale bars: $5 \mu \mathrm{m}$. 


$$
U(t)=\int_{x_{\ell 0}}^{x_{\ell 1}} u(x, t) d x .
$$

Fig. 3A,B demonstrates that this model fits our data extremely well over multiple bleachrecovery cycles (21). The fits indicate that on average the lipid mobility in our 1-D bilayers $\left(3 \pm 1 \cdot 10^{-3} \mu^{2} / s\right)$ is about three orders of magnitude lower than lipid mobility in fluid bilayers adsorbed on glass $\left(1-5 \mu^{2} / s\right)(22)$. Our diffusion coefficient values fall near the lower limit of the reported range for polyelectrolyte-supported lipid membranes $(12,13)$ (which almost always show mobility at least an order of magnitude lower than bilayers supported on glass). Notably, our 1-D bilayers showed almost two orders of magnitude lower mobility than the bilayers of the same lipid composition supported on the same polymer cushion on a flat substrate (0.1-0.2 $\left.\mu^{2} / s\right)(18)$. Higher defect density in lipid membranes on nanotubes compared to the flat bilayers might lead to obstructed diffusion (23) and to the decrease in the effective diffusion coefficient.

Interestingly, the lipid mobility in our structures can depend dramatically on the composition of the polymer cushion. A large percentage of 1-D bilayers supported on a PDDA/PSS polymer cushion showed recovery after bleaching. In these cases fluorescent images of the nanotubes before bleaching and after recovery were almost undistinguishable (insets on the Fig. 3A,B). However, the same lipid membrane supported on nanotubes with PAH/PSS polymer cushion was immobile on the time scale of our experiment, clearly showing stable bleached spots in the fluorescent image (Fig. S2). This behavior indicates that the lipid headgroups interact stronger with PAH layer than with PDDA layer (24). This effect is reminiscent of stronger coupling in PAH/PSS multilayers compared to PDDA/PSS multilayers $(25,26)$.

A critical test of the utility of the 1-D bilayers will be their ability to support membrane proteins, as shown in the Fig.1. As a proof-of-concept experiment we studied interactions of anthrax protective antigen fragment, PA63, with our lipid-coated carbon nanotubes. PA63 spontaneously and irreversibly inserts into cellular and artificial lipid membranes (27) where it forms 
a heptameric pore (28). To verify the protein ability to insert into our 1-D lipid membranes we have exposed them to a solution of PA63 labeled with Alexa633 dye. Fig. 3D shows that the protein has co-localized into several regions of the lipid-coated nanotube. We have conducted our experiments at $\mathrm{pH}=5.5$ where the interactions of the protein with the membrane are dominated by the hydrophobic forces (29), which cause the protein's insertion into the membrane. At this point we cannot verify that the protein has retained its functionality in the membrane, which must be established by further experiments. Nevertheless, our results strongly indicate that our 1-D lipids may serve as a matrix for membrane proteins.

We believe that our structures open up significant new possibilities for chemists, biologists, and materials scientists. Our system provides the opportunity to test the ultimate physical limits of bilayer self-assembly in a highly controlled and fully tunable system. Moreover, strong dependence of the lipid mobility on the properties of the underlying substrate gives us the ability to fine-tune the diffusion properties of our 1-D bilayers.

Lipid membranes provide an efficient biocompatible barrier between the nanotube surface and the surrounding solution. Reconstitution of the membrane protein channels into the 1-D bilayers should provide a robust way to control and modulate access of the small molecules to the nanotube surface. Controlled reconstitution of different types of proteins into the lipid bilayers could also enable novel biosensing schemes. Finally, lipid-coated nanotubes could be used as biocompatible probes of intracellular environments, or as structural foundation for building complex hybrid bio-inorganic nanostructures.

\section{References and Notes}

1. R. H. Baughman, A. A. Zakhidov, W. A. de Heer, Science 297, 787 (2002).

2. M. Ouyang, J. L. Huang, C. M. Lieber, Account Chem Res 35, 1018 (2002). 
3. R. J. Chen, et al., Proc. Natl. Acad. Sci. U.S.A. 100, 4984 (2003).

4. D. M. Cannon, N. Winograd, A. G. Ewing, Annu Rev Bioph Biom 29, 239 (2000).

5. J. Fritz, et al., Science 288, 316 (2000).

6. S. Q. Wang, et al., Nature Mater. 2, 196 (2003).

7. M. Zheng, et al., Nature Mater. 2, 338 (2003).

8. C. Richard, F. Balavoine, P. Schultz, T. W. Ebbesen, C. Mioskowski, Science 300, 775 (2003).

9. C. Huang, J. T. Mason, Proc. Natl. Acad. Sci. U.S.A. 75, 308 (1978).

10. M. J. O'Connell, et al., Science 297, 593 (2002).

11. A. B. Artyukhin, O. Bakajin, P. Stroeve, A. Noy, Langmuir 20, 1442 (2004).

12. T. Cassier, A. Sinner, A. Offenhauser, H. Mohwald, Colloids Surf., B 15, 215 (1999).

13. R. Kugler, W. Knoll, Bioelectrochemistry 56, 175 (2002).

14. S. Moya, et al., Macromolecules 33, 4538 (2000).

15. Materials and methods are available as supporting material on Science Online.

16. Substitution of the PAH to PDDA produces somewhat rougher but still continuous coating of 10-30 nm in diameter (Fig.S1). TEM images indicated that often our long nanotubes stretching across the grid holes were not individual nanotubes, but rather small bundles of 2-5 single wall nanotubes, which further contributed to an increased diameter of the bilayer support.

17. A. A. Brian, H. M. McConnell, Proc. Natl. Acad. Sci. U.S.A. 81, 6159 (1984). 
18. L. Q. Zhang, M. L. Longo, P. Stroeve, Langmuir 16, 5093 (2000).

19. D. Axelrod, D. E. Koppel, J. Schlessinger, E. Elson, W. W. Webb, Biophys. J. 16, 1055 (1976).

20. If the bilayers on the nanotube and the substrate are not connected, each subsequent bleaching cycle would result in recovery to a progressively lower lever, as we keep depleting the total amount of the fluorescent molecules on the nanotube. If the two bilayers are connected, the fluorescence will recover to a constant value corresponding to the equilibrium concentration of the mobile lipid molecules. The data presented on the Figure 3A,B strongly indicate that at least in some cases the two bilayers are connected.

21. If we continue the bleach-recovery cycles, we will eventually start to exhaust the lipid reservoirs at the nanotube ends. This effect is likely responsible for the drastically different quality of the model fit for the last two cycles on the Figure 3B.

22. J. F. Tocanne, L. Dupoucezanne, A. Lopez, Prog. Lipid Res. 33, 203 (1994).

23. T. V. Ratto, M. L. Longo, Biophys. J. 83, 3380 (2002).

24. Several features in the PAH chemical structure may account for stronger coupling relative to PDDA. PAH has primary amino groups which could participate in hydrogen bonding, while PDDA is a quaternary ammonium ion, which cannot form hydrogen bonds. Presence of two methyl groups attached directly to a charged nitrogen in PDDA (compared to protons in PAH) results in steric hindrance and increases separation distance between the PDDA cation center and anionic lipid groups, which reduces electrostatic binding energy. Yet another reason for weaker PDDA-lipid interaction is a lower linear charge density of PDDA compared to PAH. 
25. S. T. Dubas, J. B. Schlenoff, Langmuir 17, 7725 (2001).

26. C. Gao, S. Leporatti, S. Moya, E. Donath, H. Moehwald, Langmuir 17, 3491 (2001).

27. R. O. Blaustein, T. M. Koehler, C. R. J., A. Finkelstein, Proc. Natl. Acad. Sci. U.S.A. 86, 2209 (1989).

28. C. Petosa, R. J. Collier, K. R. Klimpel, S. H. Leppla, R. C. Liddington, Nature 385, 833 (1997).

29. T. M. Koehler, R. J. Collier, Mol Microbiol 5, 1501 (1991).

30. AA acknowledges SEGR Fellowship from LLNL. AN acknowledges LLNL LDRD funding. This work was performed under the auspices of U.S. Department of Energy by the University of California, Lawrence Livermore National Laboratory under Contract No. W-7405-Eng48. 


\section{Supplemental information}

\section{Materials and Methods}

Materials. Most of the reagents used in this study was available commercially and used as received. Activated form of recombinant anthrax protective antigen (PA63) from Bacillus anthracis (List Biolabs)was labeled with Alexa Fluor 633 dye using a protein labeling kit (Molecular Probes, Inc.). UV-Vis absorption spectra indicated degree of labeling of 0.5. Copper transmission electron microscope bare grids (2000 mesh) were obtained from Ted Pella, Inc.

Substrate and nanotube preparation. We have described the details of the nanotube synthesis and polymer adsorption in a recent publication (1). For this work we have significantly improved the rinsing procedure between adsorption steps by using simultaneous injection and withdrawal of 50-100 $\mathrm{ml}$ of liquid with two syringe pumps. Solution was continuously stirred in a 2-ml beaker during adsorption and rinsing. To minimize the possibility of nanotube damage by the surface tension, we kept the sample below liquid-air interface during the whole process while gradually exchanging solutions. We used $50 \mathrm{mM}$ polymer concentration with no added electrolyte for the layer-by-layer deposition steps.

Vesicle preparation and bilayer formation. We used ultrasonication to prepare lipid vesicles containing 1-stearoyl-2-oleoylphosphatidylserine (SOPS), 1-palmitoyl-2-oleoylphosphatidylcholine (POPC), and 2-(4,4-difluoro-5-methyl-4-bora-3a,4a- diaza-s-indacene-3-dodecanoyl)-1- hexadecanoylsn-glycero-3- phosphocholine (BODIPY-PC) in ratio 75:23:2 (SOPS:POPC:BODIPY-PC) following a previously described procedure. (2). Fresh vesicle solution was added to the reaction beaker and the grid with polymer-coated carbon nanotubes was incubated with vesicles for 40$50 \mathrm{~min}$. We previously found this time to be sufficient to reach equilibrium for bilayer formation on a flat substrate (2).

Protein adsorption. PA63 labeled with Alexa Fluor 633 dye was diluted in $10 \mathrm{mM}$ MES buffer (150 mM NaCl, $\mathrm{pH} 5.5)$ and added to a reaction beaker to a final concentration of 100 $\mathrm{nM}$. The grid with carbon nanotubes coated with 5 polymer layers and lipid bilayer was left in the protein solution for $30 \mathrm{~min}$ and then rinsed with pure buffer. 
Fluorescence Confocal Microscopy, FRAP, and TEM imaging. After all adsorption steps had been completed, we placed the grid in a thin water or buffer layer between two clean glass cover slips. Thin liquid film remaining between the cover slips could keep the grid with lipidcoated nanotubes in aqueous environment for days. Design of our laser scanning confocal microscope was described earlier. (3) All fluorescence images were collected using high NA oil-immersion objective and excitation at $488 \mathrm{~nm}$ (BODIPY-PC) and $633 \mathrm{~nm}$ (Alexa-633). All images were acquired using NanoscopeIII SPM controller (Veeco-DI). For FRAP experiments we used a high-intensity beam (ca. $50 \mu \mathrm{W}$ ) of our confocal microscope to bleach out a small region $(0.5 \mu \mathrm{m})$ on the coated nanotube. We then monitored the recovery of the fluorescence intensity in the same area using much weaker excitation intensity (ca. $1 \mu \mathrm{W}$ ). TEM images were collected on a Philips CM300 FEG transmission electron microscope, operating at 300 $\mathrm{kV}$, and utilizing a Gatan imaging filter.

\section{Supplemental Figures}

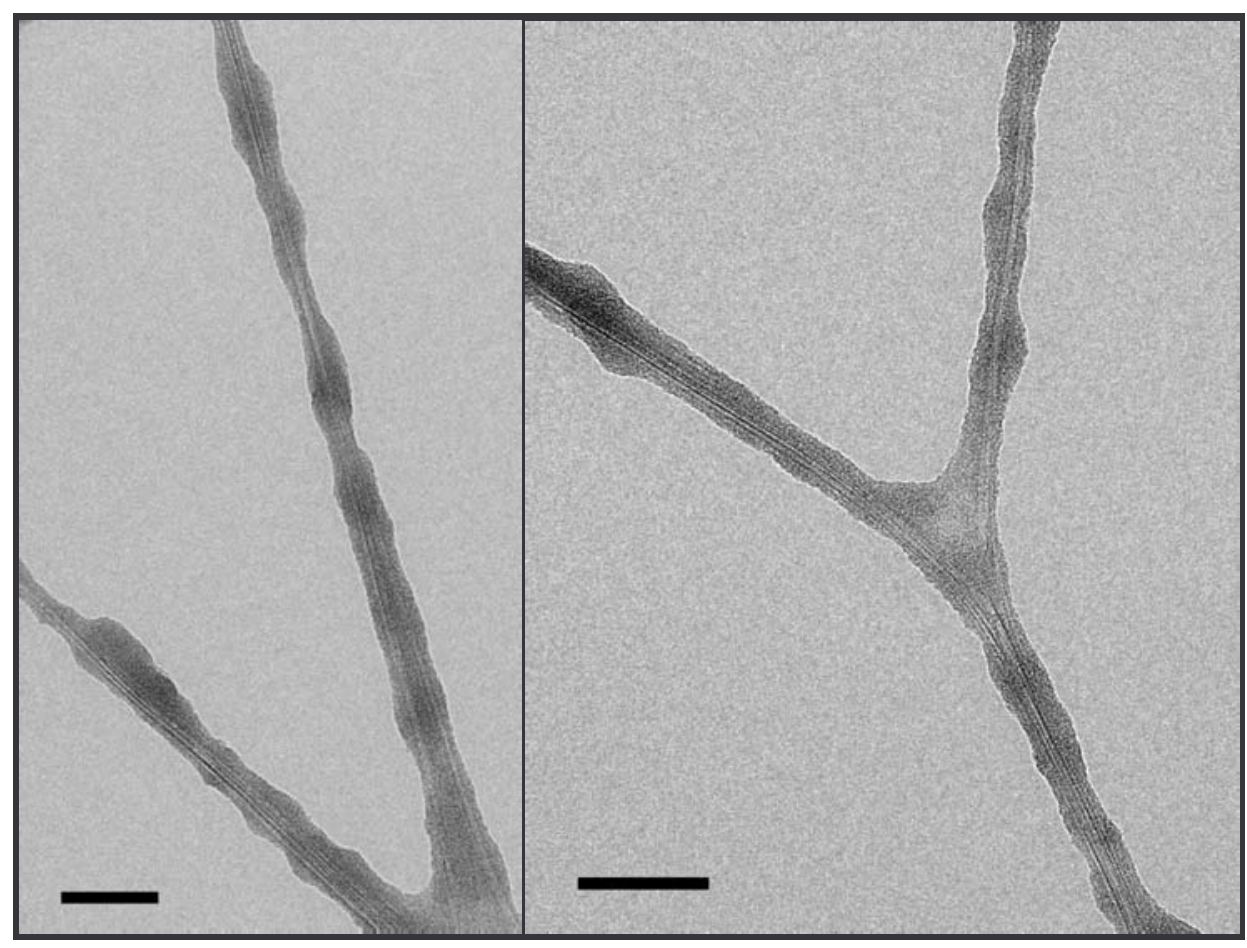

Fig. S 1: TEM images of carbon nanotubes coted with five alternating PDDA and PSS layers. Scale bars correspond to $50 \mathrm{~nm}$. 


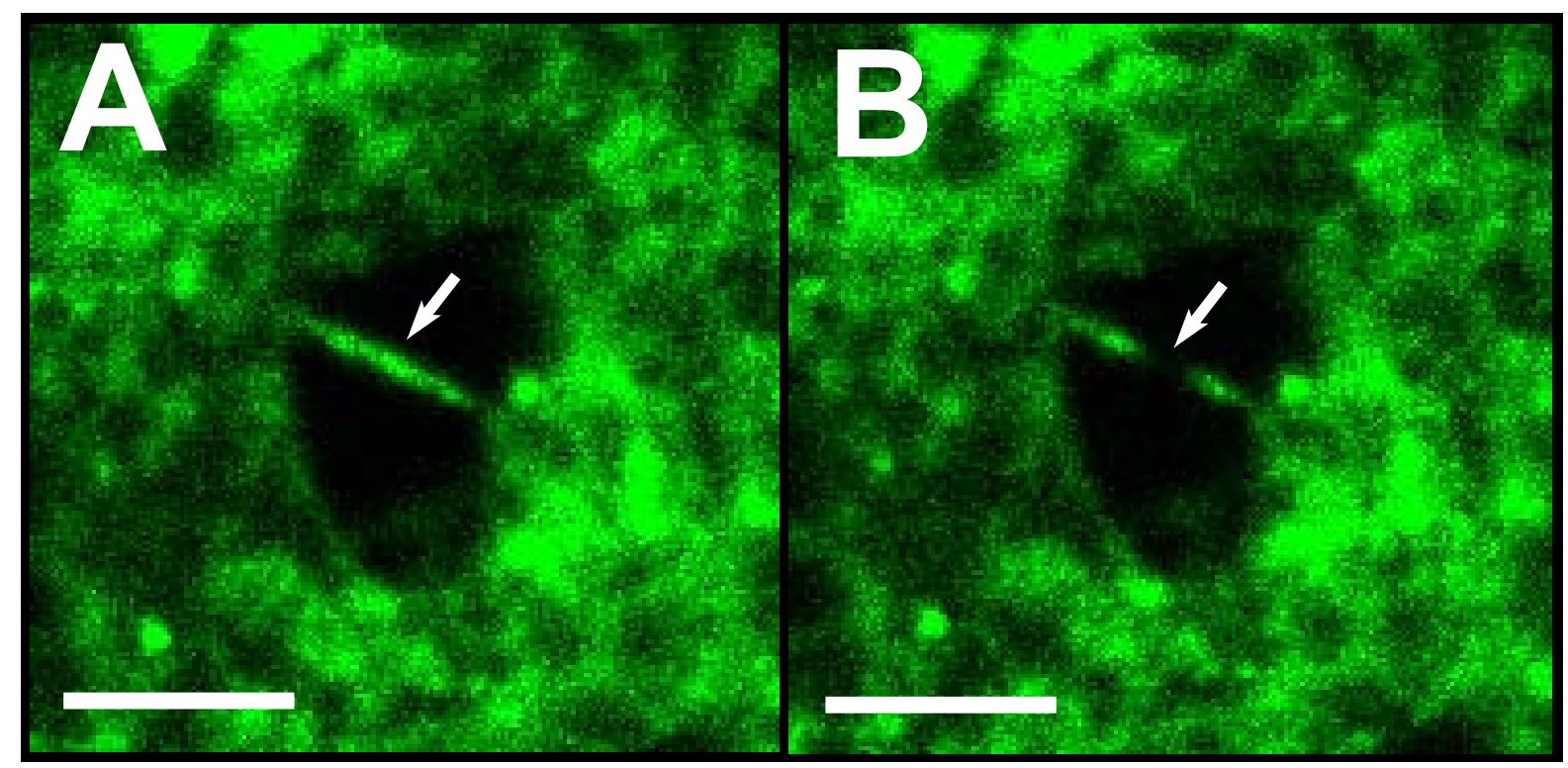

Fig. S 2: Confocal fluorescent images of TEM grids with carbon nanotubes coated with 5 PAH and PSS layers and lipid bilayer before (A) and after (B) bleaching. Arrows indicate the nanotubes. Image (B) shows a bleached region on the nanotube. Scale bars: $5 \mu \mathrm{m}$.

\section{References}

1. A. B. Artyukhin, O. Bakajin, P. Stroeve, A. Noy, Langmuir 20, 1442 (2004).

2. L. Q. Zhang, M. L. Longo, P. Stroeve, Langmuir 16, 5093 (2000).

3. A. Noy, T. R. Huser, Rev. Sci. Instruments 74, 1217 (2003). 